\title{
BMJ Open Does obstructive sleep apnoea contribute to obesity, hypertension and kidney dysfunction in children? A systematic review protocol
}

\author{
Sara Rodriguez-Lopez (D) ,,2 Stefan Palkowski, ${ }^{2,3}$ Christopher Gerdung (D) ,2,4 \\ Diana Keto-Lambert, ${ }^{2,5}$ Meghan Sebastianski, ${ }^{2,5}$ Maria Luisa Castro-Codesal (D) 2,4
}

To cite: Rodriguez-Lopez S, Palkowski S, Gerdung C, et al. Does obstructive sleep apnoea contribute to obesity, hypertension and kidney dysfunction in children? A systematic review protocol. BMJ Open 2020;10:e039342. doi:10.1136/ bmjopen-2020-039342

- Prepublication history and additional material for this paper are available online. To view please visit the journal (http:// dx.doi.org/10.1136/bmjopen2020-039342).

Received 11 April 2020 Revised 29 July 2020 Accepted 29 July 2020

Check for updates

(C) Author(s) (or their employer(s)) 2020. Re-use permitted under CC BY-NC. No commercial re-use. See rights and permissions. Published by BMJ.

For numbered affiliations see end of article.

Correspondence to

Dr Maria Luisa Castro-Codesal; castroco@ualberta.ca

\section{ABSTRACT}

Introduction Childhood obstructive sleep apnoea (OSA) is a highly prevalent disorder that may directly contribute to the development of obesity, hypertension and renal injury. Although those associations seem to be clearer in adults, studies in children have revealed conflicting results and updated synthesis of the evidence is lacking. The aim of this systematic review is to summarise the available evidence on the effect of OSA on obesity, systemic blood pressure and kidney function, to help to elucidate whether respiratory interventions to correct OSA would have the potential to improve those outcomes.

Methods and analysis A systematic literature review search was created by a medical librarian and peerreviewed by a second librarian prior to running. Ovid Medline, Ovid Embase, CINAHL via EbscoHOST, Wiley Cochrane Library and ProQuest Dissertations and Theses Global were searched on 25 February 2020. Titles and abstracts will be screened by two independent reviewers for inclusion, followed by full-text screening of relevant articles. Studies in children will be included if they report data on OSA and weight, systemic blood pressure or kidney parameters. The extracted data will be combined for analysis and the information subcategorised in groups based on outcome. Risk of bias will be determined using tools specific to study methodology and certainty of the evidence using the Grading of Recommendations, Assessment, Development and Evaluations approach. Ethics and dissemination This study will provide essential information for healthcare professionals to better understand the relationship between childhood OSA and changes in body mass index, systemic blood pressure and kidney function indicators. Our findings will be disseminated through conferences and publications. The results of this review may guide the initiation of new strategies and the development of future research studies. This research did not involve human subjects and therefore did not undergo research ethical review. PROSPERO registration number CRD42020171186.

\section{INTRODUCTION}

Childhood obstructive sleep apnoea (OSA) is characterised by recurrent events of partial or complete upper airway obstruction during sleep, resulting in disruption of normal
Strengths and limitations of this study

- Methodological and information experts were engaged to enable high-quality assessment and transparency.

- No language restrictions or time limits will be applied to the search strategy.

- Risk of bias and certainty of the evidence will be rigorously determined.

- Availability of primary studies may be limited, especially for the kidney function outcome.

- There may be low certainty of the evidence from observational studies.

ventilation and sleep patterns. ${ }^{1}$ It has become a highly prevalent disorder with rates up to $5.7 \%^{2}$ due in part to the dramatic increase in prevalence and severity of obesity in children and adolescents worldwide. ${ }^{3-7}$ In adults, it has been clearly demonstrated that OSA, the associated intermittent nocturnal hypoxemia and other sleep disturbances, has deleterious clinical consequences contributing to obesity, metabolic syndrome and cardiovascular and renal diseases ${ }^{8-12}$ with some of this evidence previously synthesised. ${ }^{13-16}$ In children, emerging data over the past few years also suggest these associations although available studies have revealed conflicting results ${ }^{17-31}$ and evidence synthesis is less available.

Childhood obesity is a widely recognised risk factor for the development of OSA ${ }^{32} 33$ and the presence of OSA has also been found to promote or aggravate obesity and associated morbidities in observational studies. ${ }^{244}{ }^{35}$ Moreover, OSA and obesity may interact and potentiate each other amplifying their adverse consequences. ${ }^{18} 3637$ Evidence showing the implications of treating obesity to ameliorate OSA has started to emerge. ${ }^{38} 39$ As well, the effect of treating OSA on obesity 
management has also been explored by more recent studies, ${ }^{30} 40-43$ justifying the need of a systematic review.

Cardiovascular morbidity of OSA in children include, among others, changes in systemic blood pressure and cardiac structure by echocardiography. ${ }^{44} 45$ Thus, several studies have demonstrated the association between OSA, high blood pressure and left ventricular hypertrophy. ${ }^{46-50}$ A recent meta-analysis synthesised the available evidence on the impact of OSA on echocardiogram parameters as a surrogate of cardiovascular disease. ${ }^{23}$ Updated highquality systematic reviews summarising the evidence on the association between OSA and changes in blood pressure are however lacking. ${ }^{51-55}$

The association between OSA and adverse renal outcomes in children is less clear than in adults ${ }^{15}$ but there are growing concerns among paediatric specialists about early initiation of kidney damage in children with OSA. Children and adolescents with OSA who are found to have high blood pressure in paediatric respiratory and sleep clinics settings are frequently referred to paediatric nephrology specialists for evaluation and management. These patients may also have subclinical or clinical renal alterations such as proteinuria, hyperfiltration or decreased glomerular filtration rate (GFR) as OSA may contribute to renal vascular dysfunction, inflammation and dysregulation of the renin-angiotensin-aldosterone system. ${ }^{56-58}$ Likewise, OSA may contribute to renal dysfunction by promoting classical risk factors for kidney disease such as obesity and diabetes mellitus. ${ }^{56}{ }^{59}$ Varlami et al did not find a significant difference in urine albuminto-creatinine ratio between children with mild OSA, moderate to severe OSA and a control group. Conversely, there is evidence of a high prevalence of sleep-disordered breathing in children with chronic kidney disease. ${ }^{60} \mathrm{~A}$ systematic review summarising the emerging evidence on the impact of OSA on renal outcomes in children will be useful for clinicians working with this population.

The aim of this systematic review is to summarise the current evidence available on the effect of childhood OSA on obesity, systemic blood pressure and kidney function. This synthesis will help to elucidate whether respiratory interventions to correct OSA will have the potential to improve those outcomes.

\section{METHODS AND ANALYSIS \\ Study design}

The protocol for this systematic review was designed in accordance with the Preferred Reporting Items for Systematic Review and Meta-Analysis Protocols guidelines. ${ }^{61}$ The study protocol has been registered in PROSPERO. We have created an advisory team that includes an expert in systematic review methodology, an information specialist, two paediatric respirologists and sleep specialists and a paediatric nephrologist to advise on the search strategy, sources of information and methodology and discussion of the results. This approach will enable a rational high-quality assessment and transparency in the report of the available evidence on the role of OSA in children and adolescents on body mass index (BMI), blood pressure changes and kidney injury.

\section{Search strategy}

Terms referring to OSA, obesity, systemic hypertension and renal function were combined for a comprehensive search strategy that captures all publications available on the topic. A previously established paediatric filter in children ${ }^{62}$ was incorporated to the search (see online supplementary appendix for the full search strategy). Study design restrictions were applied to exclude letters to the editor, comments, reviews and case reports as well as studies in animals. No language restrictions or time limits were applied to the search strategy.

\section{Information sources}

A comprehensive search strategy with described terms was built in Ovid Medline and translate into Ovid Embase, CINAHL via EbscoHOST and Wiley Cochrane Library (including the Cochrane Database of Systematic Reviews, the Cochrane Central Register of Controlled Trials, the Database of Abstracts of Reviews of Effects, the Health Technology Assessment Database and the National Health Service Economic Evaluation Database). The reference lists of the included studies will be screened to identify any relevant studies that were not detected by the search. Search of grey literature will include ProQuest Dissertations and Theses Global and published abstracts from relevant international conference proceedings such as the International Pediatric Nephrology Association conference and the Scientific Meeting of the European Society for Paediatric Nephrology.

\section{Eligibility criteria}

Participants/population

We will include studies of children and adolescents (0-18 years of age) with OSA syndrome, defined by the American Academy of Sleep Medicine (snoring and at least one other symptom of OSA including night time symptoms, daytime symptoms and physician examination) and/or the presence of an apnoea-hypopnoea index greater than 1.5 events/hours in children over 2 years of age and greater than three events/hour in children under the age of 2 years by night polysomnography. ${ }^{1763}$ Studies that contain data from both adults and children will be included if children data are reported separately.

\section{Outcomes}

Studies will be included if they report data on weight, BMI, systemic blood pressure or parameters of kidney function. We will use percentiles and/or $\mathrm{Z}$ scores to compare data regarding anthropometric measurements and percentiles for blood pressure data. Definitions of overweight/obesity will include BMI above 85/95th percentile based on Centers for Disease Control and Prevention (CDC) growth charts ${ }^{64}$ or $+1 \mathrm{SD} / 2 \mathrm{SD}$ based on WHO standards (www.who.int/growthref). CDC standards will be used for potential meta-analysis. Elevated 
blood pressure/hypertension will be defined based on current guidelines: elevated blood pressure as blood pressure above the 90th percentile for age or greater than 120/80 $\mathrm{mm} \mathrm{Hg}$ and hypertension as blood pressure above the 95th percentile or greater than $130 / 80 \mathrm{~mm}$ Hg. ${ }^{65}$ We will include studies that report renal outcomes according to the status of albuminuria/proteinuria or the estimated GFR. Chronic kidney disease will be defined as a GFR below $90 \mathrm{~mL} / \mathrm{min} / 1.73 \mathrm{~m}^{2}$ and/or albuminuria above $30 \mathrm{mg} / 24$ hours (or urine albumin to creatinine ratio greater than $30 \mathrm{mg} / \mathrm{g}$ or $3 \mathrm{mg} / \mu \mathrm{mol}$ ) for more than 3 months according to internationally accepted criteria, the Kidney Disease: Improving Global Outcomes (KDIGO) guidelines. ${ }^{66}$ The estimated GFR may be calculated by any of the accepted formulas (Schwartz or Cystatin C). Original studies will be included as far as they provide a clear description of their definitions for overweight/obesity, elevated blood pressure/hypertension or renal abnormalities.

\section{Design}

Observational and intervention studies will be considered for inclusion, including controlled before-after studies, cross-sectional studies, longitudinal observational studies, case-control studies, retrospective cohorts research and case series with seven or more cases. Case reports and case series with less than seven subjects, comments, editorials, letters and reviews will be excluded.

\section{Study records}

\section{Data management}

Records identified by the search strategy will be imported into an EndNote (V.X9, Clarivate Analytics) library for management and screening.

\section{Selection process}

Titles and abstracts of the citations identified by the search will be screened by two independent reviewers for eligibility based on our inclusion criteria. For all potentially relevant articles, full text will be retrieved and evaluated for eligibility independently by the two reviewers. Any discrepancies will be resolved through discussion between the reviewers and, if not consensus reached, a third member of the research team will be invited into the discussion. Reasons for exclusion will be recorded for articles that underwent full-text screening.

\section{Data extraction process}

Using a predesigned standardised form, one reviewer will extract the data and enter it into Microsoft Excel database (Microsoft, Redmond, Washington, USA). Verification of $20 \%$ of the data extraction will be done by a second reviewer. If agreement does not meet $80 \%$, additional verification of the data will occur, reviewing all the important data columns. One article per data set will be retained to avoid double counting of the same data published in more than one publication.

\section{Data synthesis}

The extracted data will be collected and combined for a narrative and numerical analysis of included studies. We will present a narrative description of the author, year and country of the publication, study design, participant characteristics, sample size, intervention type, control group description, outcome measures and timeframe for follow-up duration. The information will be subcategorised to include different groups based on outcome (changes in BMI/weight gain, blood pressure, kidney function parameters). Given the potential collinearity between these conditions, we will perform a stratified analysis based on the collinearity assessments of the original studies. Meta-analysis will be attempted for those studies reporting same outcomes with heterogeneity measured using the $\mathrm{I}^{2}$ statistics.

\section{Quality assessment (risk of bias and certainty of the evidence)}

Risk of bias and methodological quality will be determined for each study independently by two reviewers using tools specific to the study methodology. Quantitative studies included in our review will be assessed using the Quality Assessment Tool for Quantitative Studies ${ }^{67}$ and qualitative studies using the Critical Appraisal Skills Programme (CASP) Qualitative Research Checklist. ${ }^{68}$ The Cochrane Risk of Bias tool ${ }^{69}$ will be used to evaluate randomised controlled trials if any. Discrepancies in decisions will be resolved through discussion.

The reviewers will also use the Grading of Recommendations, Assessment, Development and Evaluations approach $^{70}$ to assess the certainty of the evidence at the outcome level: very low, low, moderate and high.

\section{Patient and public involvement \\ No patient involved.}

\section{Ethics and dissemination}

This study will provide essential information for healthcare professionals to better understand the relationship between childhood OSA and changes in BMI, systemic blood pressure and kidney function indicators. Our findings will be disseminated through conferences and publications. The results of this review may guide the initiation of new strategies and may also provide information for the development of future research studies. This research did not involve human subjects and therefore did not undergo research ethical review.

There is a clinical need to systematically summarise the emerging literature around the association between childhood OSA and deleterious outcomes such as obesity, hypertension and kidney injury. This will facilitate the work of researchers and clinicians looking after this increasing group of children and adolescents and will also help to elucidate whether potential respiratory interventions to correct OSA are helpful to ameliorate those outcomes.

Author affiliations

${ }^{1}$ Nephrology, Stollery Children's Hospital, Edmonton, Alberta, Canada 
${ }^{2}$ Pediatrics, University of Alberta Faculty of Medicine and Dentistry, Edmonton, Alberta, Canada

${ }^{3}$ Pediatrics, Stollery Children's Hospital, Edmonton, Alberta, Canada

${ }^{4}$ Respiratory Medicine, Stollery Children's Hospital, Edmonton, Alberta, Canada ${ }^{5}$ Alberta Strategy for Patient-Oriented Research (SPOR) Knowledge Translation Platform, University of Alberta, Edmonton, Alberta, Canada

Contributors SR-L, CG and MLC-C conceived the idea of the study and designed the methodology. SR-L, SP and MLC-C wrote the manuscript. MS and DK-L developed the search strategy and performed a preliminary literature review. SR-L, SP, MS, DK-L, CG and MLC-C reviewed the protocol. All authors read and approved the final manuscript.

Funding The authors have not declared a specific grant for this research from any funding agency in the public, commercial or not-for-profit sectors.

Competing interests None declared.

Patient and public involvement Patients and/or the public were not involved in the design, or conduct, or reporting, or dissemination plans of this research.

Patient consent for publication Not required.

Provenance and peer review Not commissioned; externally peer reviewed.

Open access This is an open access article distributed in accordance with the Creative Commons Attribution Non Commercial (CC BY-NC 4.0) license, which permits others to distribute, remix, adapt, build upon this work non-commercially, and license their derivative works on different terms, provided the original work is properly cited, appropriate credit is given, any changes made indicated, and the use is non-commercial. See: http://creativecommons.org/licenses/by-nc/4.0/.

\section{ORCID iDs}

Sara Rodriguez-Lopez http://orcid.org/0000-0003-0543-4855

Christopher Gerdung http://orcid.org/0000-0003-0096-3993

Maria Luisa Castro-Codesal http://orcid.org/0000-0002-1079-2502

\section{REFERENCES}

1 Loughlin GM, Brouillette RT, Brooke LJ, et al. Standards and indications for cardiopulmonary sleep studies in children. Am J Resp Crit Care 1996;153:U54.

2 Bixler EO, Vgontzas AN, Lin H-M, et al. Sleep disordered breathing in children in a general population sample: prevalence and risk factors. Sleep 2009;32:731-6.

3 Lobstein T, Baur L, Uauy R, et al. Obesity in children and young people: a crisis in public health. Obes Rev 2004;5(Suppl 1):4-85

4 Redline S, Tishler PV, Schluchter M, et al. Risk factors for sleepdisordered breathing in children. Associations with obesity, race, and respiratory problems. Am J Respir Crit Care Med 1999;159:1527-32.

5 Wing YK, Hui SH, Pak WM, et al. A controlled study of sleep related disordered breathing in obese children. Arch Dis Child 2003;88:1043-7.

6 Beebe DW, Lewin D, Zeller M, et al. Sleep in overweight adolescents: shorter sleep, poorer sleep quality, sleepiness, and sleep-disordered breathing. J Pediatr Psychol 2007;32:69-79.

7 MacLean JE, DeHaan K, Chowdhury T, et al. The scope of sleep problems in Canadian children and adolescents with obesity. Sleep Med 2018;47:44-50

8 Drager LF, Togeiro SM, Polotsky VY, et al. Obstructive sleep apnea: a cardiometabolic risk in obesity and the metabolic syndrome. J Am Coll Cardiol 2013;62:569-76.

9 Lavie L. Obstructive sleep apnoea syndrome--an oxidative stress disorder. Sleep Med Rev 2003;7:35-51.

10 Shamsuzzaman ASM, Gersh BJ, Somers VK. Obstructive sleep apnea: implications for cardiac and vascular disease. JAMA 2003;290:1906-14

11 Bradley TD, Floras JS. Obstructive sleep apnoea and its cardiovascular consequences. Lancet 2009:373:82-93.

12 Chou Y-T, Lee P-H, Yang C-T, et al. Obstructive sleep apnea: a stand-alone risk factor for chronic kidney disease. Nephrol Dial Transplant 2011;26:2244-50.

$13 \mathrm{Xu} \mathrm{S}$, Wan Y, Xu M, et al. The association between obstructive sleep apnea and metabolic syndrome: a systematic review and metaanalysis. BMC Pulm Med 2015;15:105.

14 Qian Y, Xu H, Wang Y, et al. Obstructive sleep apnea predicts risk of metabolic syndrome independently of obesity: a meta-analysis. Arch Med Sci 2016;12:1077-87.
15 Hwu D-W, Lin K-D, Lin K-C, et al. The association of obstructive sleep apnea and renal outcomes-a systematic review and metaanalysis. BMC Nephrol 2017;18:313.

16 Xie W, Zheng F, Song X. Obstructive sleep apnea and serious adverse outcomes in patients with cardiovascular or cerebrovascular disease: a PRISMA-compliant systematic review and meta-analysis. Medicine 2014;93:e336.

17 Marcus CL, Brooks LJ, Draper KA, et al. Diagnosis and management of childhood obstructive sleep apnea syndrome. Pediatrics 2012;130:e714-55.

18 Gozal D, Capdevila OS, Kheirandish-Gozal L. Metabolic alterations and systemic inflammation in obstructive sleep apnea among nonobese and obese prepubertal children. Am J Respir Crit Care Med 2008;177:1142-9.

19 Tsaoussoglou M, Bixler EO, Calhoun S, et al. Sleep-disordered breathing in obese children is associated with prevalent excessive daytime sleepiness, inflammation, and metabolic abnormalities. $J$ Clin Endocrinol Metab 2010;95:143-50.

20 Redline S, Storfer-Isser A, Rosen CL, et al. Association between metabolic syndrome and sleep-disordered breathing in adolescents. Am J Respir Crit Care Med 2007;176:401-8.

21 Canapari CA, Hoppin AG, Kinane TB, et al. Relationship between sleep apnea, fat distribution, and insulin resistance in obese children. J Clin Sleep Med 2011;7:268-73.

22 Tauman R, O'Brien LM, Ivanenko A, et al. Obesity rather than severity of sleep-disordered breathing as the major determinant of insulin resistance and altered lipidemia in snoring children. Pediatrics 2005;116:e66-73.

23 Ehsan Z, Ishman SL, Kimball TR, et al. Longitudinal cardiovascular outcomes of sleep disordered breathing in children: a meta-analysis and systematic review. Sleep 2017;40. doi:10.1093/sleep/zsx015. [Epub ahead of print: 01 Mar 2017].

24 Gozal D, Kheirandish-Gozal L. Cardiovascular morbidity in obstructive sleep apnea: oxidative stress, inflammation, and much more. Am J Respir Crit Care Med 2008;177:369-75.

25 Constantin E, McGregor CD, Cote V, et al. Pulse rate and pulse rate variability decrease after adenotonsillectomy for obstructive sleep apnea. Pediatr Pulmonol 2008;43:498-504.

26 O'Brien LM, Gozal D. Autonomic dysfunction in children with sleepdisordered breathing. Sleep 2005;28:747-52.

27 Kwok KL, Ng DKK, Cheung YF. BP and arterial distensibility in children with primary snoring. Chest 2003;123:1561-6.

28 Katz SL, MacLean JE, Hoey L, et al. Insulin resistance and hypertension in obese youth with sleep-disordered breathing treated with positive airway pressure: a prospective multicenter study. J Clin Sleep Med 2017;13:1039-47.

29 Amini Z, Kotagal S, Lohse C, et al. Effect of obstructive sleep apnea treatment on lipids in obese children. Children 2017;4. doi:10.3390/ children4060044. [Epub ahead of print: 01 Jun 2017].

30 Alonso-Álvarez ML, Terán-Santos J, Gonzalez Martinez M, et al. Metabolic biomarkers in community obese children: effect of obstructive sleep apnea and its treatment. Sleep Med 2017;37:1-9.

31 James AL, Runciman M, Burton MJ, et al. Investigation of cardiac function in children with suspected obstructive sleep apnea. $J$ Otolaryngol 2003;32:151-4

32 Li AM, So HK, Au CT, et al. Epidemiology of obstructive sleep apnoea syndrome in Chinese children: a two-phase community study. Thorax 2010;65:991-7.

33 Urschitz MS, Eitner S, Wolff J, et al. Risk factors for sleeprelated hypoxia in primary school children. Pediatr Pulmonol 2007;42:805-12.

34 Marcus CL, Brooks LJ, Draper KA, et al. Diagnosis and management of childhood obstructive sleep apnea syndrome. Pediatrics 2012;130:576-84.

35 Arens R, Muzumdar H. Childhood obesity and obstructive sleep apnea syndrome. J Appl Physiol 2010;108:436-44

36 Tan H-L, Gozal D, Kheirandish-Gozal L. Obstructive sleep apnea in children: a critical update. Nat Sci Sleep 2013;5:109-23.

37 Spruyt K, Gozal D. Mr. Pickwick and his child went on a field trip and returned almost empty handed...What we do not know and imperatively need to learn about obesity and breathing during sleep in children! Sleep Med Rev 2008;12:335-8.

38 Andersen IG, Holm J-C, Homøe P. Obstructive sleep apnea in obese children and adolescents, treatment methods and outcome of treatment - A systematic review. Int J Pediatr Otorhinolaryngol 2016;87:190-7.

39 Roche J, Isacco L, Masurier J, et al. Are obstructive sleep apnea and sleep improved in response to multidisciplinary weight loss interventions in youth with obesity? A systematic review and metaanalysis. Int J Obes 2020;44:753-70. 
40 Shine NP, Lannigan FJ, Coates HL, et al. Adenotonsillectomy for obstructive sleep apnea in obese children: effects on respiratory parameters and clinical outcome. Arch Otolaryngol Head Neck Surg 2006;132:1123-7.

41 Baldi I, Gulati A, Lorenzoni G, et al. Public health implications of obstructive sleep apnea burden. Indian J Pediatr 2014;81(Suppl 1):55-62.

42 Alajmi M, Schmalz L, Nettel-Aguirre A, et al. Continuous positive airway pressure requirements in three groups of children: obese, non-obese and children with Down syndrome. Sleep 2012;35:A387.

43 Castro-Codesal ML, Dehaan K, Bedi PK, et al. Long-term benefits in sleep, breathing and growth and changes in adherence and complications in children using noninvasive ventilation. Can J Resp Crit Care Sleep Med 2020;4:115-23.

44 Marcus CL, Greene MG, Carroll JL. Blood pressure in children with obstructive sleep apnea. Am J Respir Crit Care Med 1998;157:1098-103.

45 Miman MC, Kirazli T, Ozyurek R. Doppler echocardiography in adenotonsillar hypertrophy. Int J Pediatr Otorhinolaryngol 2000;54:21-6.

46 Kang K-T, Chiu S-N, Weng W-C, et al. Analysis of 24-hour ambulatory blood pressure monitoring in children with obstructive sleep apnea: a hospital-based study. Medicine 2015;94:e1568.

47 Kang K-T, Chiu S-N, Weng W-C, et al. Comparisons of office and 24-hour ambulatory blood pressure monitoring in children with obstructive sleep apnea. J Pediatr 2017;182:e172:177-83.

48 Kirk V, Midgley J, Giuffre M, et al. Hypertension and obstructive sleep apnea in Caucasian children. World J Cardiol 2010;2:251-6.

49 DelRosso LM, King J, Ferri R. Systolic blood pressure elevation in children with obstructive sleep apnea is improved with positive airway pressure use. J Pediatr 2018;195:e101:102-7.

50 Hanlon CE, Binka E, Garofano JS, et al. The association of obstructive sleep apnea and left ventricular hypertrophy in obese and overweight children with history of elevated blood pressure. J Clin Hypertens 2019;21:984-90.

51 Patinkin ZW, Feinn R, Santos M. Metabolic consequences of obstructive sleep apnea in adolescents with obesity: a systematic literature review and meta-analysis. Child Obes 2017;13:102-10.

52 Teo DT, Mitchell RB. Systematic review of effects of adenotonsillectomy on cardiovascular parameters in children with obstructive sleep apnea. Otolaryngol Head Neck Surg 2013;148:21-8.

53 Zintzaras E, Kaditis AG. Sleep-disordered breathing and blood pressure in children: a meta-analysis. Arch Pediatr Adolesc Med 2007;161:172-8.
54 Kwok K-li, Ng DK, Chan C-hong. Cardiovascular changes in children with snoring and obstructive sleep apnoea. Ann Acad Med Singapore 2008;37:715-21.

$55 \mathrm{Ng}$ DK, Chan C, Chow AS, et al. Childhood sleep-disordered breathing and its implications for cardiac and vascular diseases. $J$ Paediatr Child Health 2005;41:640-6.

56 Turek NF, Ricardo AC, Lash JP. Sleep disturbances as nontraditional risk factors for development and progression of CKD: review of the evidence. Am J Kidney Dis 2012;60:823-33.

57 Ozkok A, Kanbay A, Odabas AR, et al. Obstructive sleep apnea syndrome and chronic kidney disease: a new cardiorenal risk factor. Clin Exp Hypertens 2014;36:211-6.

58 Hanly PJ, Ahmed SB. Sleep apnea and the kidney: is sleep apnea a risk factor for chronic kidney disease? Chest 2014;146:1114-22.

59 Varlami V, Malakasioti G, Alexopoulos El, et al. Low-grade albuminuria in children with obstructive sleep apnea. J Sleep Res 2013;22:289-94.

60 Amin R, Sharma N, Al-Mokali K, et al. Sleep-disordered breathing in children with chronic kidney disease. Pediatr Nephrol 2015;30:2135-43.

61 Moher D, Shamseer L, Clarke M, et al. Preferred reporting items for systematic review and meta-analysis protocols (PRISMA-P) 2015 statement. Syst Rev 2015;4:1.

62 Desmeules R. Filter to Retrieve pediatric articles in the OVIDMedline database. Available: http://guides.library.ualberta.ca/aecontent.php? pid $=448005$

63 Kothare SV, Rosen CL, Lloyd RM, et al. Quality measures for the care of pediatric patients with obstructive sleep apnea. J Clin Sleep Med 2015;11:385-404.

64 Kuczmarski RJ, Ogden CL, Guo SS, et al. 2000 CDC growth charts for the United States: methods and development. Vital Health Stat 11 2002;11:1-190.

65 Flynn JT, Kaelber DC, Baker-Smith CM, et al. Clinical practice guideline for screening and management of high blood pressure in children and adolescents. Pediatrics 2017;140. doi:10.1542/ peds.2017-1904. [Epub ahead of print: 21 Aug 2017].

66 KDIGO. 2012 clinical practice guidelines for the evaluation and management of chronic kidney disease 2013;3:5.

67 Thomas H. Quality assessment tool for quantitative studies. effective public health practice project, 2003. Available: https://www.ephpp. $\mathrm{ca} /$ quality-assessment-tool-for-quantitative-studies/

68 UK C. Critical appraisal skills programme qualitative research checklist, 2016. Available: http://www.casp-uk.net/\#!casp-tools-checklists/c18f8

69 Higgins JPT, Altman DG, Gøtzsche PC, et al. The Cochrane collaboration's tool for assessing risk of bias in randomised trials. BMJ 2011;343:d5928.

70 Guyatt G, Oxman AD, Akl EA, et al. GRADE guidelines: 1. Introduction-GRADE evidence profiles and summary of findings tables. J Clin Epidemiol 2011;64:383-94. 